\author{
Taro Takahara \\ Thomas Kwee \\ Satoshi Kibune \\ Reiji Ochiai \\ Tetsuro Sakamoto \\ Tetsu Niwa \\ Marc Van Cauteren \\ Peter Luijten
}

\section{Whole-body MRI using a sliding table and repositioning surface coil approach}

Received: 18 May 2009

Revised: 19 July 2009

Accepted: 22 August 2009

Published online: 8 December 2009

C) The Author(s) 2009.

This article is published with open access at Springerlink.com

Electronic supplementary material The online version of this article (doi:10.1007/ s00330-009-1674-1) contains supplementary material, which is available to authorized users.

T. Takahara $(\bowtie) \cdot$ T. Kwee $\cdot$ T. Niwa .

P. Luijten

Department of Radiology,

University Medical Centre Utrecht,

Heidelberglaan 100 ,

P.O. Box 85500,3508 GA

Utrecht, The Netherlands

e-mail: tarorin@rr.iij4u.or.jp

Tel.: +31-30-2506687

Fax: +31-30-2542531

S. Kibune

Department of Radiology,

Koga Hospital 21,

Kurume, Fukuoka, Japan

R. Ochiai

Department of Radiology,

Fukuoka Hospital,

Fukuoka, Fukuoka, Japan

\author{
T. Sakamoto \\ Philips Healthcare Japan, \\ Shinagawa, Japan \\ T. Niwa \\ Department of Radiology, \\ Kanagawa Children's Medical Center, \\ Yokohama, Japan \\ M. Van Cauteren \\ Philips Healthcare, Asia Pacific, \\ Shinagawa, Japan
}

\begin{abstract}
Objective: To introduce and assess a new way of performing whole-body magnetic resonance imaging (MRI) using a non-integrated surface coil approach as available on most clinical MRI systems worldwide. Methods: Ten consecutive asymptomatic subjects prospectively underwent whole-body MRI for health screening. Whole-body MRI included T1-, T2- and diffusionweighted sequences, and was performed using a non-integrated surface coil to image four different stations without patient repositioning. The four separately acquired stations were merged, creating seamless coronal
\end{abstract}

whole-body T1-, T2- and diffusionweighted images. Anatomical alignment, image quality at the boundaries of adjacent stations, and overall image quality of all stations were qualitatively assessed. Results: The average time $( \pm \mathrm{SD})$ taken to change the surface coil from one station to the next station was $53.8( \pm 7.1) \mathrm{s}$. The average total extra examination time $\pm \mathrm{SD}$ was $2 \min 41.4 \mathrm{~s}$ $( \pm 15.3 \mathrm{~s})$. Anatomical alignment, image quality at the boundaries of adjacent stations, and overall image quality of all stations of T1-, T2- and diffusion-weighted whole-body MRI were overall graded as "good" to "excellent". Conclusion: This study shows that a time-efficient and highquality whole-body MRI examination can easily be performed by using a non-integrated sliding surface coil approach.

Keywords Sliding coil - Wholebody . Diffusion weighted imaging . Cancer screening $\cdot$ Magnetic resonance imaging

\section{Introduction}

Magnetic resonance imaging (MRI) offers high spatial resolution and excellent soft-tissue contrast, making it an ideal tool for the detection of parenchymal and osseous lesions. Furthermore, MRI does not use any potentially harmful radiation. MRI may therefore be an attractive technique for the detection and characterisation of disease throughout the entire body [1,2]. Previously, whole-body MRI was hampered by severe limitations. Repositioning of the patient and the surface coils to image the separate stations of the whole-body MRI examination led to 
exceedingly long examination times. Furthermore, anatomical alignment between separately imaged stations was not maintained using this approach. This limitation has been overcome by the development of the sliding table platform, which allows sequential movement of the patient through the bore of the magnet without patient repositioning, while signal is received by either an integrated body coil or by a (more recently introduced) whole-body surface coil design [1-3]. A whole-body surface coil design yields superior signal-to-noise ratio (SNR) and spatial resolution compared with an integrated transmit-receive body coil. Moreover, the use of surface coils allows for parallel imaging, which may be necessary for imaging time reduction and for acquiring certain types of MRI sequences, such as diffusion-weighted MRI (DWI). Of note, DWI has recently been implemented in a whole-body MRI examination and is a potentially powerful tool for the detection and characterisation of various oncological and non-oncological lesions $[4,5]$. Thus, the use of a wholebody surface coil design is preferred to perform a wholebody MRI examination. The interest in the clinical application of whole-body MRI is rapidly increasing, as is well illustrated by the large number of publications in this field over the past few years [6-17]. Furthermore, whole-body MRI may be an excellent alternative to wholebody positron emission tomography (PET)/computed tomography (CT) $[6-8,10,12,13]$, given its wider availability [18] and the lack of ionising radiation [19]. However, most MR systems in routine clinical practice may not yet be equipped with an integrated whole-body surface coil design. Moreover, for a large number of these systems, an easily available upgrade towards fully integrated whole-body surface coil technology may not be available (yet). On the other hand, non-integrated surface coils (some of which are capable of parallel imaging) are widely available. These surface coils, however, have only a limited anatomical coverage. Nevertheless, it may still be possible to perform a whole-body MRI examination using a non-integrated surface coil. The aim of this study was, therefore, to introduce and qualitatively assess a new and easy approach to performing a time-efficient and highquality whole-body MRI examination (including DWI) using a non-integrated surface coil.

\section{Materials and methods}

\section{Asymptomatic subjects}

Institutional review board approval was waived because MRI examinations were performed as part of routine patient care. Ten consecutive asymptomatic subjects [five men and five women; mean age, 61.6 years (range, 5279 years); mean height, $160.2 \mathrm{~cm}$ (range, 154-167 cm); mean weight $55.6 \mathrm{~kg}$ (range, 44-62 kg); mean body mass index $21.6 \mathrm{~kg} / \mathrm{m}^{2}$ (range, $18.3-24.2 \mathrm{~kg} / \mathrm{m}^{2}$ )], who under- went whole-body MRI for health screening, were prospectively included. Subjects with a general contraindication for MRI (including implanted pacemaker and claustrophobia) were excluded from participation.

\section{Table preparation}

In order to perform a whole-body MRI examination using a non-integrated surface coil, four spacers (each with a height of $5 \mathrm{~cm}$ ) were placed on the original patient table. Subsequently, an additional table platform (Tabletop extender, Philips Healthcare, Best, The Netherlands) was mounted on top of these four spacers. In this way, sufficient space was created to freely move the lower part of a surface coil over a distance of approximately $110 \mathrm{~cm}$ along the $\mathrm{z}$ axis, without the need to reposition the subject who is lying on top of the additional table platform (Fig. 1). As a tradeoff, the use of spacers and an additional table platform reduced the available vertical bore diameter by $6.5 \mathrm{~cm}$.

\section{MRI}

All subjects were examined by 1.5-T MRI (Achieva, Philips Healthcare, Best, The Netherlands), using a fourelement phased-array surface coil (SENSE body, Philips Healthcare, Best, The Netherlands), in the head-first position. Four different anatomical stations (station $1=$ head/neck; station 2 = chest; station $3=$ abdomen; station 4 = pelvis) were separately imaged, using T1-, T2- and diffusion-weighted sequences. Geometries among the different stations of the T1-, T2- and diffusion-weighted sequences were coupled using software implemented in the standard operating console (Geolinks, Philips Healthcare, Best, The Netherlands). After imaging one station, the operator entered the examination room, moved the surface coil to the next station, and repositioned the centre of the coil at the isocentre of the magnet (three station changes in total), without repositioning the subject (Movie clip 1). In this way, three-dimensional (3D) alignment among the imaged stations was maintained. Of note, the operator was outside the examination room during scanning. The positions and overlaps of the four stations in DWI are shown in Fig. 2. To maintain sufficient SNR in the periphery of the field of view (FOV) of each station in the z-direction, a $3-\mathrm{cm}$ overlap between two adjacent stations was applied. Furthermore, a 7-cm overlap was applied between stations 1 and 2 , in order to maintain robust fat suppression in the neck/shoulder region, an area which may suffer considerably from magnetic field heterogeneity. Because of the gradient design, no corrections for nonlinear gradient behaviour had to be applied.

T1-weighted gradient-echo imaging was performed with the following sequence parameters per station: repetition time (TR)/echo time (TE), 187/2.3 (in-phase) and 4.6 (out- 

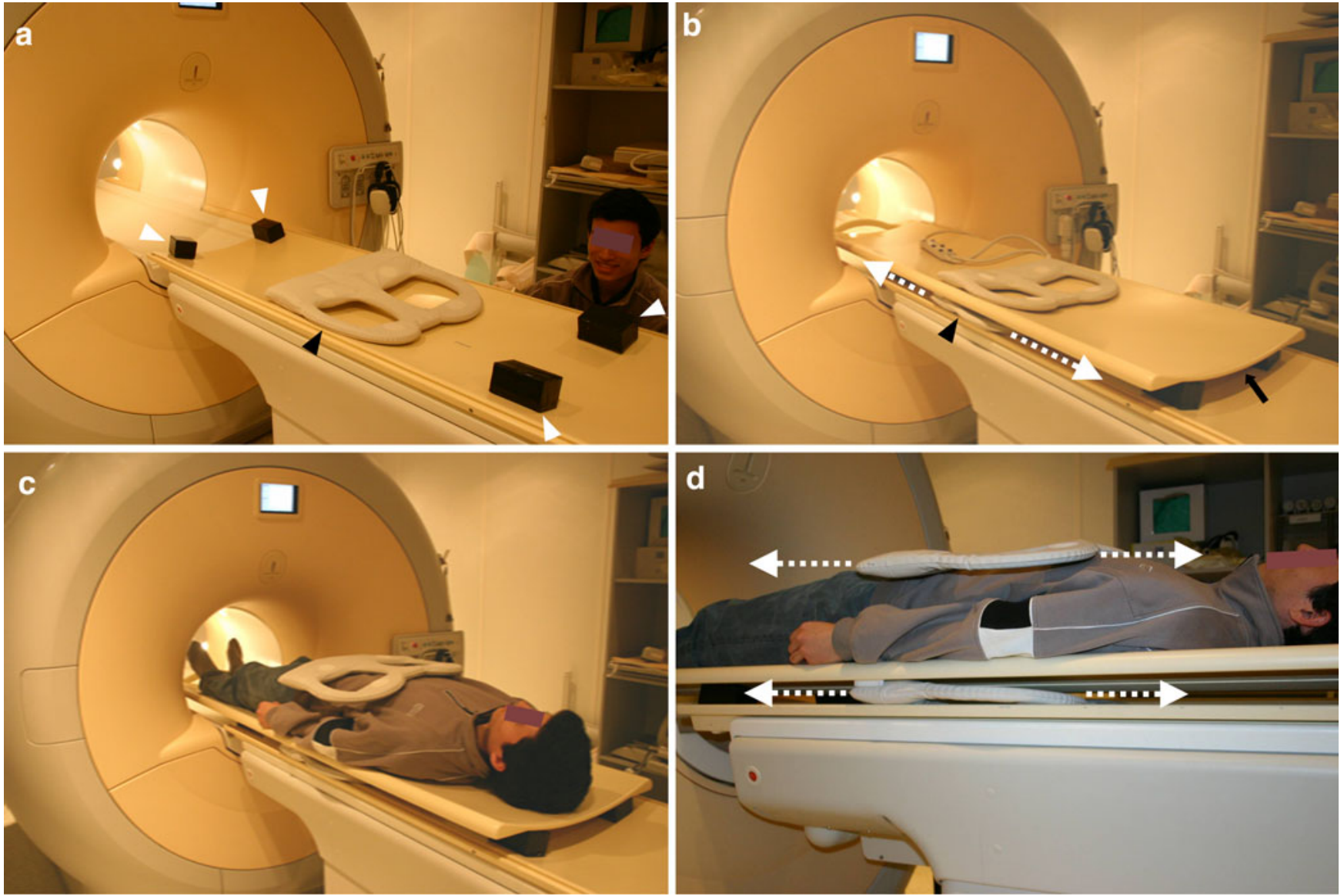

Fig. 1 Table preparation for whole-body MRI using a sliding surface coil approach. a Spacers (white arrowheads) are placed on top of the original patient table to create space for the lower part of the surface coil (black arrowhead). b An additional table platform is mounted on top of the spacers (arrow). The lower part of the surface

coil (black arrowhead) can be moved freely below the additional table platform (dashed arrows). c, d Patient is lying on top of the additional table platform; the surface coil can be moved freely without patient repositioning (dashed arrows)

of-phase) ms; image acquisition in the coronal plane; slice thickness/gap, 7.0/1.0 mm; number of slices, 25; FOV, $400 \mathrm{~mm}^{2}$; acquisition matrix, 224; one signal average; imaging percentage (phase encode reduction), 65\%; actual pixel size $1.79 \times 2.74 \mathrm{~mm}^{2}$, reconstructed pixel size, $1.25 \times$ $1.25 \mathrm{~mm}^{2}$; image acquisition under (end-inspiratory) breath-holding; total imaging time, $28 \mathrm{~s}$ per station.

T2-weighted turbo spin-echo imaging was performed with the following sequence parameters per station: TR/ TE, $1,000 / 82 \mathrm{~ms}$; image acquisition in the coronal plane; slice thickness/gap, 7.0/1.0 mm; number of slices, 25; FOV, $400 \mathrm{~mm}^{2}$; acquisition matrix, 320; one signal average; imaging percentage (phase encode reduction), 96\%; half image factor, 0.525 ; parallel imaging [sensitiviy encoding (SENSE)] factor, 2.0; actual pixel size, $1.25 \times$ $1.31 \mathrm{~mm}^{2}$; reconstructed pixel size, $0.78 \times 0.78 \mathrm{~mm}^{2}$; image acquisition under (end-inspiratory) breath-holding; total imaging time, $25 \mathrm{~s}$ per station.

Diffusion-weighted single-shot echo-planar imaging (EPI) was performed in the transverse plane with slice

overlaps in station 1 (head/neck), because station 1 is relatively more prone to image distortion. No slice overlaps were used for DWI of stations 2-4 (chest, abdomen, and pelvis). Station 1: TR/TE/inversion time, 5,320/68/180 ms; slice thickness/gap, 4.0/-1.0 mm; number of packages, 2; number of slices, 75; four signal averages; total imaging time, $191 \mathrm{~s}$. Stations 2-4: TR/TE/inversion time, 9,249/68/180 ms; slice thickness/gap, 4.0/0 mm; number of packages, 1; number of slices, 65 ; five signal averages; total imaging time, $212 \mathrm{~s}$ per station. The remaining parameters for DWI were the same in all stations: FOV, $400 \mathrm{~mm}^{2}$; rectangular FOV, 70\%; acquisition matrix, 160; motion probing gradients in three orthogonal axes; number of $b$ values, $2\left(0\right.$ and $\left.1,000 \mathrm{~s} / \mathrm{mm}^{2}\right)$; imaging percentage (phase encode reduction), $80 \%$; half image factor, 0.6 ; parallel imaging (SENSE) factor, 2.0 (of note, to enable parallel imaging, each station needed separate preparatory imaging); EPI factor, 47; actual pixel size, $2.50 \times 3.19 \mathrm{~mm}^{2}$; reconstructed pixel size, $1.56 \times 1.56 \mathrm{~mm}^{2}$; image acquisition under free breathing. 


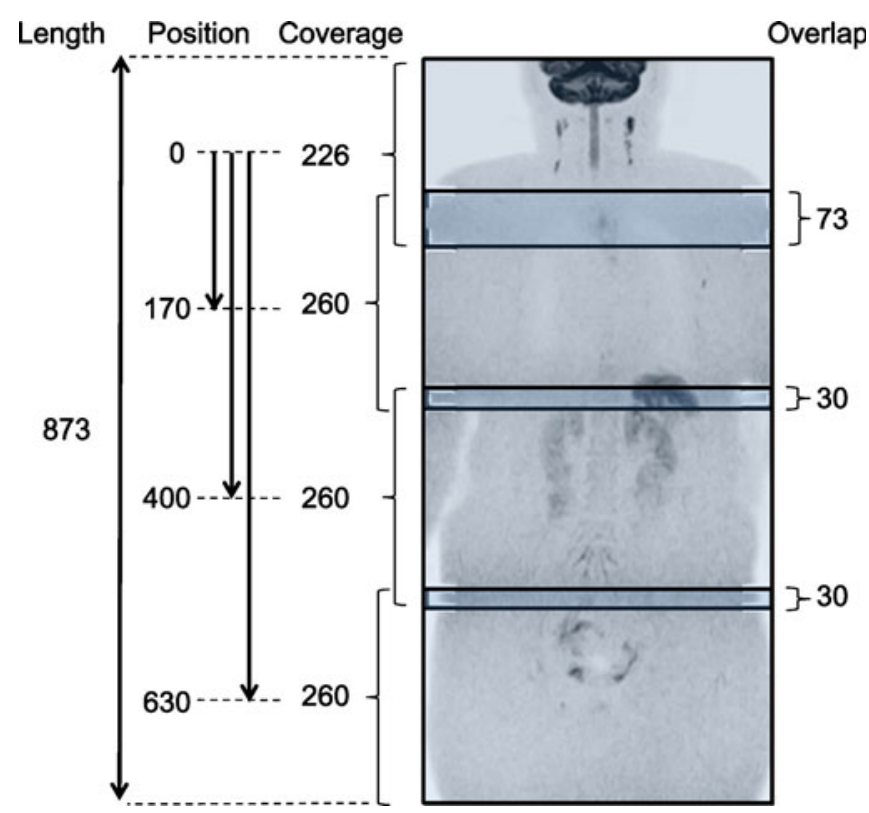

Fig. 2 Positions and overlaps of the four stations in DWI, with explanatory measures (in $\mathrm{mm}$ ). Imaging length of DWI was $87.3 \mathrm{~cm}$, and aimed to cover the body from the level of the ear to the inguinal region

Diffusion-weighted images were coronally reformatted, with a slice thickness/gap of 5.0/0 mm, and displayed using grey-scale inversion. Subsequently, the four separately acquired stations were merged by software implemented in the standard operating console (MobiView, Philips Healthcare, Best, The Netherlands), for each of the coronal T1weighted, coronal T2-weighted and coronal reformatted DWI datasets. In this way, seamless coronal whole-body T1-weighted, T2-weighted and diffusion-weighted images were created, each covering the area from at least the neck to the inguinal area (Fig. 3).

\section{Data analysis}

Time required to change the surface coil from one station to the next station was measured for each of the three station changes, in each asymptomatic subject. Average time \pm SD to change the surface coil from one station to the next station and average total extra examination time \pm SD were calculated.

Subsequently, the coronal whole-body T1-, T2- and diffusion-weighted images were reviewed by two independent board-certified radiologists (blinded by the author, with 20 and 12 years' experience in MRI interpretation). Anatomical alignment between adjacent stations in each sequence was assessed using a five-point grading scale $(1=$ very poor, anatomical alignment completely lacking; $2=$ poor, anatomical alignment differs by more than $20 \mathrm{~mm}$; $3=$ moderate, anatomical alignment differs by more than $5 \mathrm{~mm}$, but less than $20 \mathrm{~mm} ; 4=$ good, anatomical alignment differs by less than $5 \mathrm{~mm} ; 5=$ excellent, exact anatomical alignment). Furthermore, both image quality at the boundaries of adjacent stations and overall image quality of all stations in each sequence were assessed using a four-point grading scale $(1=$ inadequate image quality with marked artefacts; 2 = adequate image quality with diagnostically relevant artefacts; 3 = good image quality with slight, diagnostically irrelevant artefacts; 4 = excellent image quality without artefacts).

Median scores regarding anatomic alignment between adjacent stations, image quality at the boundaries of different adjacent stations, and overall image quality of all different stations were calculated for each sequence. The Kruskal-Wallis test was used to test for overall equality of medians in each data group. When significant differences occurred, independent samples were compared by using the Mann-Whitney $U$-test. Differences were considered significant when $P$ values were less than 0.05 . Statistical analyses were executed using Statistical Package for the Social Sciences (SPSS) software version 16.0 (SPSS, Chicago, Ill., USA).

\section{Results}

All subjects tolerated this whole-body MRI protocol well (including breath-holding for T1- and T2-weighted imaging), without any claustrophobic events. Average total scan time $\pm \mathrm{SD}$ was $38 \mathrm{~min} 10 \mathrm{~s} \pm 2 \min 16 \mathrm{~s}$. Average effective room time $\pm \mathrm{SD}$ was $41 \mathrm{~min} 8 \mathrm{~s} \pm 2$ min $18 \mathrm{~s}$. Average time \pm SD to change the surface coil from one station to the next station was $53.8 \pm 7.1 \mathrm{~s}$ (range, 35-67 s). Average total extra examination time \pm SD for imaging four stations (corresponding to three coil repositionings) was $2 \mathrm{~min}$ $41.4 \mathrm{~s} \pm 15.3 \mathrm{~s}$ (range, $2 \mathrm{~min} 16 \mathrm{~s}$ to $3 \mathrm{~min} 1 \mathrm{~s}$ ).

Median scores regarding anatomical alignment were 5 (excellent) between all stations on all sequences, and were not significantly different from each other, for both observers (Table 1).

Median scores regarding image quality were 4 (excellent) at all station boundaries of T1- and T2-weighted images, and were not significantly different from each other, for both observers (Table 2). However, although median scores regarding image quality at the boundaries of different stations of diffusion-weighted images were 3 (good) or 4 (excellent), they were significantly different from each other, for both observers (Table 2). Pairwise comparisons revealed that the quality of diffusion-weighted images at the boundaries of stations 1 and 2 (head/neck and chest) was significantly better ( $P \leq 0.001$ for both observers) than that at the boundaries of stations 2 and 3 (chest and abdomen) and stations 3 and 4 (abdomen and pelvis), without any significant differences between the other stations.

Median scores regarding overall image quality were either 3 (good) or 4 (excellent) for all stations of all sequences and for both observers, but these scores were 
Fig. 3 Representative examples of whole-body T1-weighted (a), T2-weighted (b) and diffusionweighted (c) images, acquired using the sliding surface coil approach, in an asymptomatic subject without abnormal findings (case 2). Note that anatomical alignment and image quality at the boundaries between the head/neck and chest stations, and between the boundaries of the abdominal and pelvic stations are good to excellent, whereas those at the boundaries between the chest and abdominal stations are moderate to good (arrowheads)
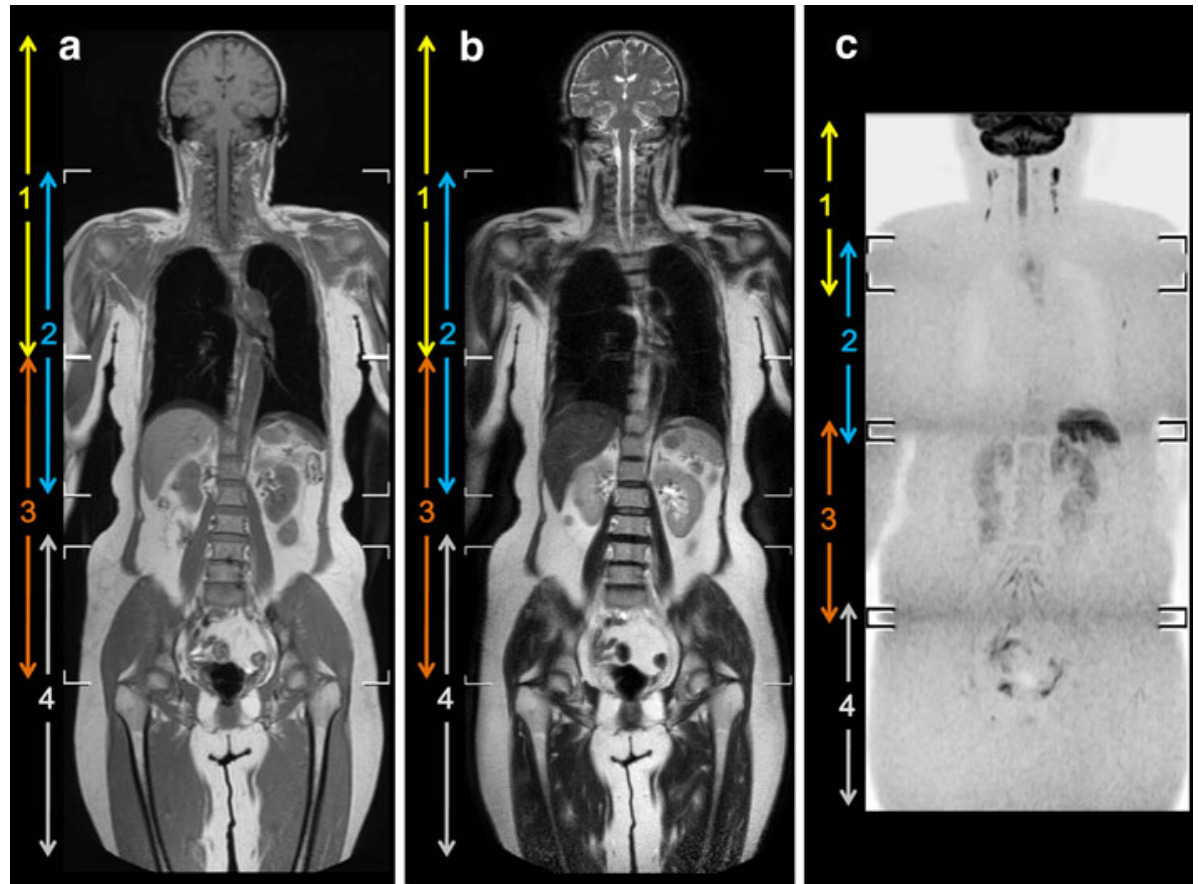

almost always significantly different from each other (Table 3). Pairwise comparisons first revealed that the overall quality of T1-weighted images of stations 1 (head/ neck), 3 (abdomen), and 4 (pelvis) was significantly better $(P=0.030)$ than that of station 2 (chest) for observer 1 , without any significant differences between the other stations. Findings of observer 2 were slightly different; the overall quality of T1-weighted images of station 1 (head/ neck) was significantly better $(P<0.03)$ than that of all other stations, and the overall quality of T1-weighted images of station 4 (pelvis) was significantly better $(P=$ 0.004 ) than that of station 3 (abdomen), without any significant differences between other stations. Second, overall quality of T2-weighted images of stations 1 (head/ neck), 3 (abdomen), and 4 (pelvis) were significantly better $(P=0.012$ for observer 1 and $P<0.001$ for observer 2$)$ than that of station 2 (chest), without any significant differences between the other stations. Third, although median scores regarding overall quality of diffusion-weighted images of all stations were not significantly different from each other for observer 1, this was the case for observer 2; pairwise comparisons revealed that overall quality of diffusionweighted images of station 1 (head/neck) was significantly better $(P=0.012)$ than that of all other stations, without any significant differences between the other stations.

A representative example of a whole-body T1-weighted, a T2-weighted and a diffusion-weighted image using a nonintegrated sliding surface coil approach can be seen in Fig. 3.

One subject had a high suspicion of lung cancer because of a high signal intensity area in the lung at DWI, which was histopathologically confirmed. No abnormal findings were detected in any of the other nine subjects.

Table 1 Overall comparison of scores regarding anatomical alignment between different stations

\begin{tabular}{llllll}
\hline Sequence & Observer & \multicolumn{2}{l}{$P$ vedian score (range) } & Stations 3↔4 \\
\cline { 2 - 5 } & & Stations $1 \leftrightarrow 2$ & Stations $2 \leftrightarrow 3$ & $5(-)$ & 1.000 \\
\hline T1-weighted & 1 & $5(-)$ & $5(-)$ & $5(4-5)$ & 1.000 \\
T2-weighted & 2 & $5(4-5)$ & $5(4-5)$ & $5(-)$ & 1.000 \\
DWI & 1 & $5(-)$ & $5(-)$ & $5(-)$ & 1.000 \\
& 2 & $5(-)$ & $5(-)$ & $5(-)$ & 1.000 \\
\hline
\end{tabular}

Scoring system: 1 = very poor, anatomical alignment completely lacking; 2 = poor, anatomical alignment differs by more than $20 \mathrm{~mm} ; 3=$ moderate, anatomical alignment differs by more than $5 \mathrm{~mm}$, but less than $20 \mathrm{~mm}$; 4 = good, anatomical alignment differs by less than $5 \mathrm{~mm}$; $5=$ excellent, exact anatomical alignment 
Table 2 Comparison of scores regarding image quality at the boundaries of different stations

\begin{tabular}{|c|c|c|c|c|c|}
\hline \multirow[t]{2}{*}{ Sequence } & \multirow[t]{2}{*}{ Observer } & \multicolumn{3}{|c|}{ Median score (range) } & \multirow[t]{2}{*}{$P$ value } \\
\hline & & Stations $1 \leftrightarrow 2$ & Stations $2 \leftrightarrow 3$ & Stations $3 \leftrightarrow 4$ & \\
\hline \multirow[t]{2}{*}{ T1-weighted } & 1 & $4(-)$ & $4(-)$ & $4(-)$ & 1.000 \\
\hline & 2 & $4(-)$ & $4(-)$ & $4(-)$ & 1.000 \\
\hline \multirow[t]{2}{*}{ T2-weighted } & 1 & $4(-)$ & $4(-)$ & $4(-)$ & 1.000 \\
\hline & 2 & $4(-)$ & $4(-)$ & $4(-)$ & 1.000 \\
\hline \multirow[t]{2}{*}{ DWI } & 1 & $4(-)$ & $3(2-4)$ & $3(-)$ & $<0.001$ \\
\hline & 2 & $4(-)$ & $3(2-4)$ & $3(2-4)$ & $<0.001$ \\
\hline
\end{tabular}

Scoring system: 1 = inadequate image quality with marked artifacts; $2=$ adequate image quality with diagnostically relevant artifacts; $3=$ good image quality with slight, diagnostically irrelevant artifacts; $4=$ excellent image quality without artifacts

\section{Discussion}

Whole-body MRI has evolved into a clinically feasible technique over the past few years [1-17]. In MRI, and especially in whole-body MRI, there is always a trade-off between image quality and imaging time. Regardless of the type of MRI sequence used and the imaging speed itself, handling of the patient through the MRI system to obtain a whole-body examination has always been a major issue. Streamlining this process will yield both a reduction in total examination time and an increase in image quality. Previously, whole-body MRI was performed by repeatedly repositioning the patient in order to image the separate stations of the whole-body MRI examination; this significantly increased total examination time. Furthermore, anatomical alignment between separately imaged stations was not maintained using this approach; acquired images of separate stations had to be adjusted and aligned manually to create the visually appealing whole-body image [3]. Then the moving table platform was introduced, allowing for sequential movement of the patient through the bore of the magnet during imaging; patient repositioning was no longer required and total examination time was reduced. Furthermore, the slice selection gradients match exactly at each station, as a result of which anatomical alignment between separate stations is maintained [3].
Post-processing software capable of automatically aligning the different stations and fusing them into one seamless whole-body image has been developed; this significantly reduced the time required for processing the images after the examination. However, signal in whole-body MRI was still acquired using an integrated body coil, which yields inferior SNR and spatial resolution compared with a surface coil [3]. Recently, whole-body surface coil technology (combining a large number of seamlessly integrated coil elements and independent radiofrequency channels) was introduced, which offers whole-body coverage and overcomes the problem of compromised image quality inherent to signal reception using an integrated body coil $[1,2]$. Furthermore, a whole-body surface coil design allows for parallel imaging, which may be necessary for imaging time reduction and for DWI $[4,5]$. On the other hand, non-integrated surface coils such as those still being used on most of the currently installed systems are widely available, yield excellent image quality and allow for parallel imaging. However, their limited anatomical coverage may be a practical issue.

The present study shows that it is feasible to perform a time-efficient and high-quality whole-body MRI examination (including DWI), using a non-integrated surface coil. Using the sliding surface coil approach, average extra examination time was only less than 3 min (161.4 s) for

Table 3 Comparison of scores regarding overall image quality of each station

\begin{tabular}{|c|c|c|c|c|c|c|}
\hline \multirow[t]{2}{*}{ Sequence } & \multirow[t]{2}{*}{ Observer } & \multicolumn{4}{|c|}{ Median score (range) } & \multirow[t]{2}{*}{$P$ value } \\
\hline & & Station 1 & Station 2 & Station 3 & Station 4 & \\
\hline \multirow[t]{2}{*}{ T1-weighted } & 1 & $4(-)$ & $4(2-4)$ & $4(-)$ & $4(-)$ & 0.005 \\
\hline & 2 & $4(-)$ & $3(2-4)$ & $3(-)$ & $4(3-4)$ & $<0.001$ \\
\hline \multirow[t]{2}{*}{ T2-weighted } & 1 & $4(-)$ & $3.5(2-4)$ & $4(-)$ & $4(-)$ & 0.001 \\
\hline & 2 & $4(-)$ & $3(2-4)$ & $4(2-4)$ & $4(3-4)$ & $<0.001$ \\
\hline \multirow[t]{2}{*}{ DWI } & 1 & $4(-)$ & $4(-)$ & $4(3-4)$ & $4(-)$ & 0.392 \\
\hline & 2 & $4(-)$ & $3.5(3-4)$ & $3(2-4)$ & $3.5(3-4)$ & 0.012 \\
\hline
\end{tabular}

Scoring system: 1 = inadequate image quality with marked artifacts; 2 = adequate image quality with diagnostically relevant artifacts; 3 = good image quality with slight, diagnostically irrelevant artifacts; $4=$ excellent image quality without artifacts 
acquiring four separate stations. Furthermore, anatomical alignment between adjacent stations, image quality at the boundaries of adjacent stations, and overall image quality of all stations were graded as "good" to "excellent" overall. The quality of diffusion-weighted images at the boundaries of the chest and abdominal station, and at the boundaries of the abdominal and pelvic stations were relatively poorer (although still graded as "good" overall). In addition, the quality of T1-, T2- and diffusion-weighted images of the head/neck station tended to be better than that of the chest, abdominal, and pelvic stations (although still graded as "good" overall). This finding can be explained by the fact that the chest, abdominal, and pelvic regions are more prone to breathing artefacts (i.e. the continuously changing position of the diaphragm, paradiaphragmatic, and abdominal organs during image acquisition and between the two imaging procedures may result in slight image blurring) than the head/neck region. It should be noted that this problem is most likely not related to the sliding surface coil approach, and would also have occurred when using a whole-body surface coil design. Nonetheless, it may potentially hinder image interpretation and decrease lesion conspicuity.

Using the sliding surface coil approach, sufficient space was created to freely move the lower part of a surface coil over a distance of approximately $110 \mathrm{~cm}$ along the z-axis. However, true whole-body imaging was not performed, because the upper extremities were just outside the FOV, while the lower extremities were not examined at all. Nevertheless, the region from the head to the pelvis, the most essential part of the body when performing wholebody imaging studies (e.g. in cases of oncological staging) could easily be covered in all subjects.

Another relative drawback of the proposed sliding surface coil approach is narrowing of the bore diameter in the vertical direction by $6.5 \mathrm{~cm}$ because of the use of spacers and an additional table platform. Especially in the Western world, where the prevalence of obesity and the number of large-sized patients is increasing, this may be an issue [20]. Furthermore, narrowing of the bore diameter may increase the risk of claustrophobic events, although all subjects in the present study tolerated the whole-body MRI examination well. Another drawback is the need to prepare the MRI table. However, this requires only a negligible amount of extra time (less than $5 \mathrm{~min}$ ). Additionally, in the present study, a surface coil with a relatively small anatomical coverage (24 cm in the z-axis) was used. The use of a surface coil with larger anatomical coverage may even further decrease extra examination time, because fewer coil repositionings will be required. For example, a surface coil with $48 \mathrm{~cm}$ coverage (which is currently widely available for routine clinical use) allows up to $96 \mathrm{~cm}$ of imaging along the z-axis (with maintenance of anatomical alignment), with only one coil repositioning. Eventually, it is expected that the availability of wholebody surface coil designs will increase and that they will become the method of choice when performing a wholebody MRI examination. Until then, the sliding surface coil approach is an excellent alternative for performing a whole-body MRI examination.

A relative study limitation is the fact that the slice thickness of acquired coronal T1- and T2-weighted images (slice thickness/gap of 7.0/1.0 mm) did not match that of coronally reformatted diffusion-weighted images (slice thickness/gap of 5.0/0.0 mm). However, it would be impractical to acquire thinner T1- and T2-weighted slices, because this would considerably improve scan time and decrease SNR. Furthermore, the acquired axial diffusionweighted dataset was reformatted into thin coronal slices rather than into thicker slices, because the former theoretically allows better lesion evaluation.

In conclusion, the present study showed that a timeefficient, high-quality whole-body MRI examination can easily be performed by using a sliding surface coil approach.

Acknowledgements We thank Ms. Naoko Tsutsumi, Ms. Satomi Yano, and Ms. Asaka Obuchi for technical assistance in scanning. This work was funded (T.T.) by Nederandse Organisatie voor Wetenschappelijk Onderzoek (NWO) and the Japanese Society of Magnetic Resonance in Medicine (JSMRM).

Open Access This article is distributed under the terms of the Creative Commons Attribution Noncommercial License which permits any noncommercial use, distribution, and reproduction in any medium, provided the original author(s) and source are credited.

\section{References}

1. Lauenstein TC, Semelka RC (2005) Whole-body magnetic resonance imaging. Top Magn Reson Imaging $16: 15-20$
2. Lauenstein TC, Semelka RC (2006) Emerging techniques: whole-body screening and staging with MRI. J Magn Reson Imaging 24:489-498

3. Hargaden G, O'Connell M, Kavanagh E, Powell T, Ward R, Eustace S (2003) Current concepts in whole-body imaging using turbo short tau inversion recovery MR imaging. AJR Am J Roentgenol 180:247-252
4. Takahara T, Imai Y, Yamashita T, Yasuda S, Nasu S, Van Cauteren M (2004) Diffusion weighted whole body imaging with background body signal suppression (DWIBS): technical improvement using free breathing, STIR and high resolution 3D display. Radiat Med 22(4):275-282 
5. Kwee TC, Takahara T, Ochiai R, Nievelstein RA, Luijten PR (2008) Diffusion-weighted whole-body imaging with background body signal suppression (DWIBS): features and potential applications in oncology. Eur Radiol 18:1937-1952

6. Yi CA, Shin KM, Lee KS et al (2008) Non-small cell lung cancer staging: efficacy comparison of integrated PET/ CT versus 3.0-T whole-body MR imaging. Radiology 248:632-642

7. Ohno Y, Koyama H, Onishi Y et al (2008) Non-small cell lung cancer: whole-body MR examination for M-stage assessment - utility for wholebody diffusion-weighted imaging compared with integrated FDG PET/CT. Radiology 248:643-654

8. Plathow C, Aschoff P, Lichy MP et al (2008) Positron emission tomography/ computed tomography and whole-body magnetic resonance imaging in staging of advanced nonsmall cell lung cancer-initial results. Invest Radiol 43:290-2977
9. Brauck K, Zenge MO, Vogt FM et al (2008) Feasibility of whole-body MR with T2- and T1-weighted real-time steady-state free precession sequences during continuous table movement to depict metastases. Radiology 246:910916

10. Pfannenberg C, Aschoff P, Schanz S et al (2007) Prospective comparison of $18 \mathrm{~F}$-fluorodeoxyglucose positron emission tomography/computed tomography and whole-body magnetic resonance imaging in staging of advanced malignant melanoma. Eur J Cancer 43:557-564

11. Mürtz P, Krautmacher C, Träber F, Gieseke J, Schild HH, Willinek WA (2007) Diffusion-weighted whole-body MR imaging with background body signal suppression: a feasibility study at 3.0 Tesla. Eur Radiol 17:3031-3037

12. Lichy MP, Aschoff P, Plathow $\mathrm{C}$ et al (2007) Tumor detection by diffusionweighted MRI and ADC-mapping-initial clinical experiences in comparison to PET-CT. Invest Radiol 42:605-613

13. Schmidt GP, Schoenberg SO, Schmid R et al (2007) Screening for bone metastases: whole-body MRI using a 32channel system versus dual-modality PET-CT. Eur Radiol 17:939-949

14. Schmidt GP, Baur-Melnyk A, Herzog P et al (2005) High-resolution wholebody magnetic resonance image tumor staging with the use of parallel imaging versus dual-modality positron emission tomography-computed tomography: experience on a 32-channel system. Invest Radiol 40:743-753
15. Schlemmer HP, Schäfer J, Pfannenberg C et al (2005) Fast whole-body assessment of metastatic disease using a novel magnetic resonance imaging system: initial experiences. Invest Radiol 40:64-71

16. Lauenstein TC, Goehde SC, Herborn CU et al (2004) Whole-body MR imaging: evaluation of patients for metastases. Radiology 233:139-148

17. Antoch G, Vogt FM, Freudenberg LS et al (2003) Whole-body dual-modality PET/CT and whole-body MRI for tumor staging in oncology. JAMA 290:3199-3206

18. Ahmad MN, Zafar AM, Nadeem N (2009) Where there is no PET/CT. Eur J Radiol 70:463-454

19. Huang B, Law MW, Khong PL (2009) Whole-body PET/CT scanning: estimation of radiation dose and cancer risk. Radiology 251:166-174

20. Uppot RN, Sahani DV, Hahn PF, Gervais D, Mueller PR (2007) Impact of obesity on medical imaging and image-guided intervention. AJR Am J Roentgenol 188:433-440 